\title{
Acknowledgment to reviewers—2015
}

Published online: 19 January 2016

(C) Springer-Verlag Berlin Heidelberg 2016

The editors and the publisher gratefully acknowledge the excellent work provided by our reviewers over the past year. The quality of the journal is definitely dependent upon the review process. The work performed by our reviewers is essential for the increasing interest of our journal within the plastic surgery arena in Europe and elsewhere. We have a common interest in making the European Journal of Plastic Surgery an even better one as well as a leading plastic surgery journal. We wish you a happy and prosperous 2016 !

\author{
Horacio Mayer \\ Jorg Bahm \\ Paolo Persichetti \\ Alexandra Condé Green \\ Virve Koljonen \\ Cenk Demirdover \\ Steven Lo \\ Mustafa Keskin \\ Henry Svensson \\ Andrew Batchelor \\ Gustav Gulyas \\ Richard van Merkesteyn \\ Norbert Pallua \\ Horacio Zenha \\ Camilo J. Roldán \\ J.M.R. Serra-Renom \\ Yaron Har-Shai \\ Bernardo Hontanilla \\ Horacio Costa \\ Rytis Rimdeika \\ Arash Momeni \\ Tahsin Oğuz Acartürk \\ Adam Cohen \\ Damien Grinsell \\ Donald Hudson \\ Patricia Eadie \\ Frank Farbod
}

George Psaras
Oren Lapid
Petros Spyriounis
Maurizio Governa
Jason Toppi
Kongkrit Chaiyasate
Hebert Lamblet
Dae-Hyun Lew
Filip Stillaert
Gottfried Wechselberger
George Zambacos
Yves Harder
Sven-Olof Wikstrom
Tommasangelo Petitti
Stephan Spendel
Halil Canter
Pablo Antonio Ysunza
Li Dong
Lydia Ferreira
Nicole Lindenblatt
Rado Zic
Fabio Santanelli di Pompeo
Hans-Eberhard Schaller
Mateusz Zachara
Paul van Zuijlen
Marco Fraccalvieri
Jeff Topf

Joseph Molnar

Gudjon Gunnarsson

Aleh Stasievich

Andreas Yiacoumettis

Ulrich Kneser

Stefan Riml

Steffen Eisenhardt

Shahram Ghotb Sajjadi

Stamatis Sapountzis

Yves Goffart

Holger Bannasch

Nenad Stepic

Mustafa Raşid Toksöz

Carlos Oscar Uebel

Daniel Kalbermatten

Mahendra Daya

Alfonso Barrera

Krishna Vyas

Kristina Klinto

Moustapha Hamdi

Umar Khan

Marcus Lehnhardt

Salvatore Giordano

Sammy Al-Benna

Raymund Horch

Ravinder Jarial

Mario Goisis 
Jens Sørensen

Adriana Tuttolomondo

Burt Brent

Assaf Zeltzer

Anita Mohan
Denis Codazzi

Eduardo Montag

Mohamed Ellabban

Stephen Goldie

Umut Ersoy
Tommaso Agostini

Salvatore D'Arpa

Warren Rozen 